\title{
Current status of ALPACA for exploring sub-PeV gamma-ray sky in Bolivia
}

\section{T. Sako ${ }^{a}$ on behalf of the ALPACA Collaboration}

(a complete list of authors can be found at the end of the proceedings)

${ }^{a}$ Institute for Cosmic Ray Research, University of Tokyo, Kashiwa, Chiba 277-8582, Japan

E-mail: sako@icrr.u-tokyo.ac.jp

The Andes Large area PArticle detector for Cosmic ray physics and Astronomy (ALPACA) project is now constructing an air shower array near the Chacaltaya mountain at the altitude of 4,740 $\mathrm{m}$ in Bolivia. An $82,800 \mathrm{~m}^{2}$ surface area will be covered by 401 scintillating counters of $100 \mathrm{~cm} \times 100 \mathrm{~cm} \times 5 \mathrm{~cm}$ (thick). In addition to this conventional surface array, underground muon detectors covering a total of $3,600 \mathrm{~m}^{2}$ allow precise identification of muon components in air showers. Using this array, ALPACA will explore the sub-PeV gamma-ray sky first time in the Southern hemisphere. The prime target of ALPACA is to reveal PeV cosmic-ray accelerators presumably existing in the galactic plane including the galactic center. A prototype array ALPAQUITA consisting of 97 surface counters and a $900 \mathrm{~m}^{2}$ muon detector is under construction and is planned to start data taking in 2021 . The next extension to the 200 counters and $3,600 \mathrm{~m}^{2}$ muon detectors named ALPACA (half) is scheduled for 2022. In this contribution, a general introduction to ALPACA, the current status of ALPAQUITA with its infrastructure, and the extension plan after 2022 are presented.

$37^{\text {th }}$ International Cosmic Ray Conference (ICRC 2021)

July 12th - 23rd, 2021

Online - Berlin, Germany 


\section{Introduction}

Identification of PeV-particle-accelerating objects called PeVatrons is one of the most critical topics in astroparticle physics. Gamma-ray emissions above $100 \mathrm{TeV}$ are expected from PeVatrons because typically $10 \%$ of parent energy is carried by leading $\pi^{0}$,s, which immediately decays into two gamma rays, when a $\mathrm{PeV}$ particle interacts with the interstellar medium. Because of a low gamma-ray flux at such an energy range, high duty cycle and wide field of view observations using air shower arrays are advantageous to the Cherenkov observations. On the other hand, traditional air shower arrays did not have sufficient power to reject enormous background showers produced by isotropic hadronic cosmic rays. In the last few years, high-altitude air shower arrays with improved particle identification power have succeeded in detecting $>100 \mathrm{TeV}$ gamma rays in the northern hemisphere. Following the first detection from the Crab nebula by the Tibet AS $\gamma$ collaboration [1], HAWC [2] and LHAASO [3] also reported the detections. Very recent detections from Cygnus cocoon by HAWC [4], SNR G106.3 +2.7 by Tibet AS $\gamma$ [5], and 12 discrete sources by LHAASO [6] open a new era of the highest energy gamma-ray astronomy. Furthermore, the detection of diffuse sub-PeV gamma rays by Tibet $\mathrm{AS} \gamma$ [7] arose various insights on the nature of cosmic-ray particles in our Galaxy (ex. [8] [9]).

As expected from significant $\gamma \gamma$ attenuation at this energy range, observed $>100 \mathrm{TeV}$ gamma rays are concentrated on the galactic plane. Naturally, the number of such energetic sources is expected more near the galactic center region that can not be observed from the current northern observatories. As reported by the H.E.S.S. Galactic plane survey [10], many objects are known to emit gamma rays at $\mathrm{TeV}$ energy range and extend their spectra above $10 \mathrm{TeV}$. One of the most exciting targets is the galactic center from where H.E.S.S. reported hard (index of 2.3) gamma-ray emission without a break up to $40 \mathrm{TeV}$ [11]. Observations of the galactic center provide a unique opportunity to study particle accelerations around supermassive black holes. Higher diffuse gamma-ray flux than the northern hemisphere is predicted [12] because of a possibly higher cosmic-ray density and abundant molecular clouds as a target of gamma-ray producing interaction. Diffuse gamma-ray observations in the southern hemisphere are exciting topics to know the history of cosmic-ray acceleration and its propagation.

Because of the richness of the southern sub-PeV gamma-ray astronomy and based on the established technique, the Andes Large area PArticle detector for Cosmic ray physics and Astronomy (ALPACA) collaboration is planning to construct a new air shower array in Bolivia near the Chacaltaya mountain. The collaboration is now constructing a prototype array covering about $25 \%$ of the full ALPACA area, called ALPAQUITA. The operation of ALPAQUITA will start after the construction of the first underground muon detector (MD) in 2021. In 2022 an extension to full surface coverage but with a half detector density and three more MDs, namely ALPACA (half), is scheduled. Detailed performance based on the dedicated MC simulations of these arrays is presented separately in this conference [13] [14]. In this paper, a general introduction to the ALPACA project with the current construction status and plan in the coming years are reported. 
Table 1: Specifications of the original ALPACA design. Details in the different construction stages are summarized in Tab.2.

$\begin{array}{ll}\text { Location } & \text { Chacaltaya plateau, Bolivia } \\ \text { Longitude } & 68^{\circ} 08^{\prime} \mathrm{W} \\ \text { Latitude } & 16^{\circ} 23^{\prime} \mathrm{S} \\ \text { Altitude } & 4,740 \mathrm{~m} \text { a.s.l. }\left(572 \mathrm{~g} / \mathrm{cm}^{2}\right) \\ \text { Surface area } & 82,800 \mathrm{~m}^{2} \\ \text { Underground muon detector area } & 5,400 \mathrm{~m}^{2} \\ \text { Number of surface detector } & 401\left(1 \mathrm{~m}^{2} \times 5 \mathrm{~cm}^{t} \text { each }\right) \\ \text { Energy resolution }(100 \mathrm{TeV}) & 20 \% \\ \text { Angular resolution }(100 \mathrm{TeV}) & 0.2^{\circ}\end{array}$

\section{Outline of ALPACA}

The site of ALPACA as summarized in Tab.1 is located on the way from the La Paz city to the Chacaltaya mountain in Bolivia. At this latitude, a significant fraction of the southern sky is visible. The galactic center located at the declination $\delta=-29^{\circ}$ culminates at the zenith angle of $13^{\circ}$. Annual exposures with zenith angle limits up to $45^{\circ}$ and $60^{\circ}$ are plotted in Fig. 1 as a function of declination. In this calculation, reduction factor $\cos \left(\theta_{z e n}\right)$ of the geometrical area viewed from a zenith angle $\theta_{z e n}$ is taken into account. The $45^{\circ}$ limit in the zenith angle shows a good analysis performance [13] [14], while dedicated studies are needed to analyze more inclined showers.

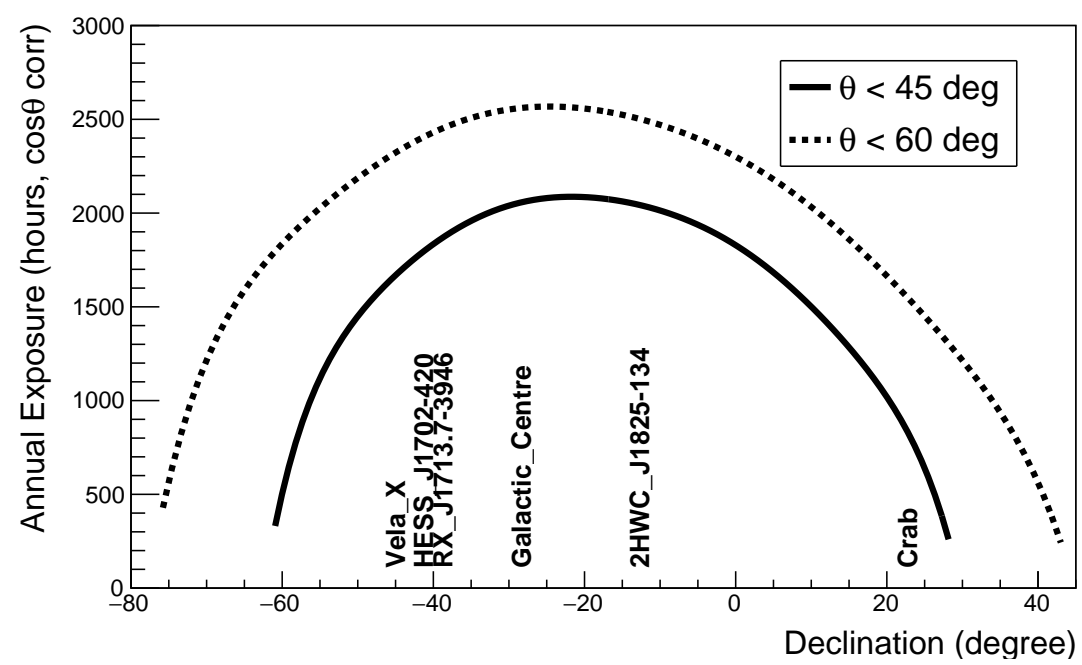

Figure 1: Annual exposure from the ALPACA site as a function of declination. Thick solid (dotted) line assumes zenith angle coverage up to $45^{\circ}\left(60^{\circ}\right)$. Names of major TeV gamma-ray sources are indicated at their declinations.

The array consists of two main components; surface air shower detectors (SDs) and underground 
MDs. The number of the SDs and the number of MDs at the different stages of ALPACA are summarized in Tab.2, together with the construction year and surface coverage. The array layouts at different stages are shown in Fig.2. Each SD comprises of $1 \mathrm{~m}^{2} \times 5 \mathrm{~cm}$ thick plastic scintillator, with a $5 \mathrm{~mm}$ thick lead plate on top, viewed by a fast timing photomultiplier tube (PMT) and a wide dynamic range PMT. The MDs are water Cherenkov detectors constructed underground with $2 \mathrm{~m}$ soil overburden equivalent to 16 radiation lengths. A single MD contains 16 cells, each having a $56 \mathrm{~m}^{2}$ area with $1.5 \mathrm{~m}$ deep clean water viewed by a PMT of 20" diameter, as shown in Fig.3. Most of the electromagnetic particles are absorbed in the soil before reaching this water pool, while a muon with energy above $1 \mathrm{GeV}$ can penetrate into the water and generate Cherenkov lights. After the lights are scattered on the wall, on average, 24 photo electrons on PMT are detected per a single muon. The detection of pure muon signals enables a $99.9 \%$ rejection of hadronic showers while keeping $80 \%$ of gamma-ray initiated showers at $100 \mathrm{TeV}$. The concept and essential performance were intensively studied by the TibetAS $\gamma$ collaboration [15] [16] and validated by their recent successful observations, as discussed in Sec.1.

Table 2: ALPACA staging

\begin{tabular}{ccccc} 
Stage & $\begin{array}{c}\text { Construction } \\
\text { Year }\end{array}$ & $\begin{array}{c}\text { Surface coverage } \\
(\text { Number of SDs })\end{array}$ & $\begin{array}{c}\text { Number of MDs } \\
(1=16 \text { cells })\end{array}$ & Reference \\
\hline ALPAQUITA & 2021 & $18,450 \mathrm{~m}^{2}(97)$ & 1 & {$[13]$} \\
ALPACA (half) & 2022 & $82,800 \mathrm{~m}^{2}(200)$ & 4 & {$[14]$} \\
ALPACA (HD) & & $82,800 \mathrm{~m}^{2}(401)$ & $4+$ & \\
Mega ALPACA & $2028+$ & $1,000,000 \mathrm{~m}^{2}(1500)$ & 50 &
\end{tabular}

\section{Status of ALPACA}

\subsection{ALPAQUITA}

The prototype array ALPAQUITA is now under construction. The array layout is shown in Fig.2 (left) as the enclosed area at the left-bottom corner. In this layout (and ALPACA High Density array) the interval of the SDs is $15 \mathrm{~m}$. Though the size of the array is small, ALPAQUITA is not only an engineering prototype. As discussed in [13] some interesting objects are already within a sensitivity of ALPAQUITA.

Figure 4 shows photographs of the SDs, cable drain, and central electronics hut. Cable drains are already reinforced with bricks and concrete and ready to arrange cables as seen in Fig.4 (right). In the central hut, the data acquisition room, and workshop, kitchen, bedroom and bathroom are available for scientists and engineers to stay during construction and maintenance works. Electric power is supplied from a nearby substation of the DeLapaz company to the Chacaltaya observatory, and a $200 \mathrm{~m}$ branch line is extended to the ALPACA site located on the way. Long-distance Wifi is available to/from the nearby El Alto town.

Some geophysical studies are under way to decide the water source. A promising location is at $900 \mathrm{~m}$ from the ALPACA site, where a small lake exists, and underground water is available with a 


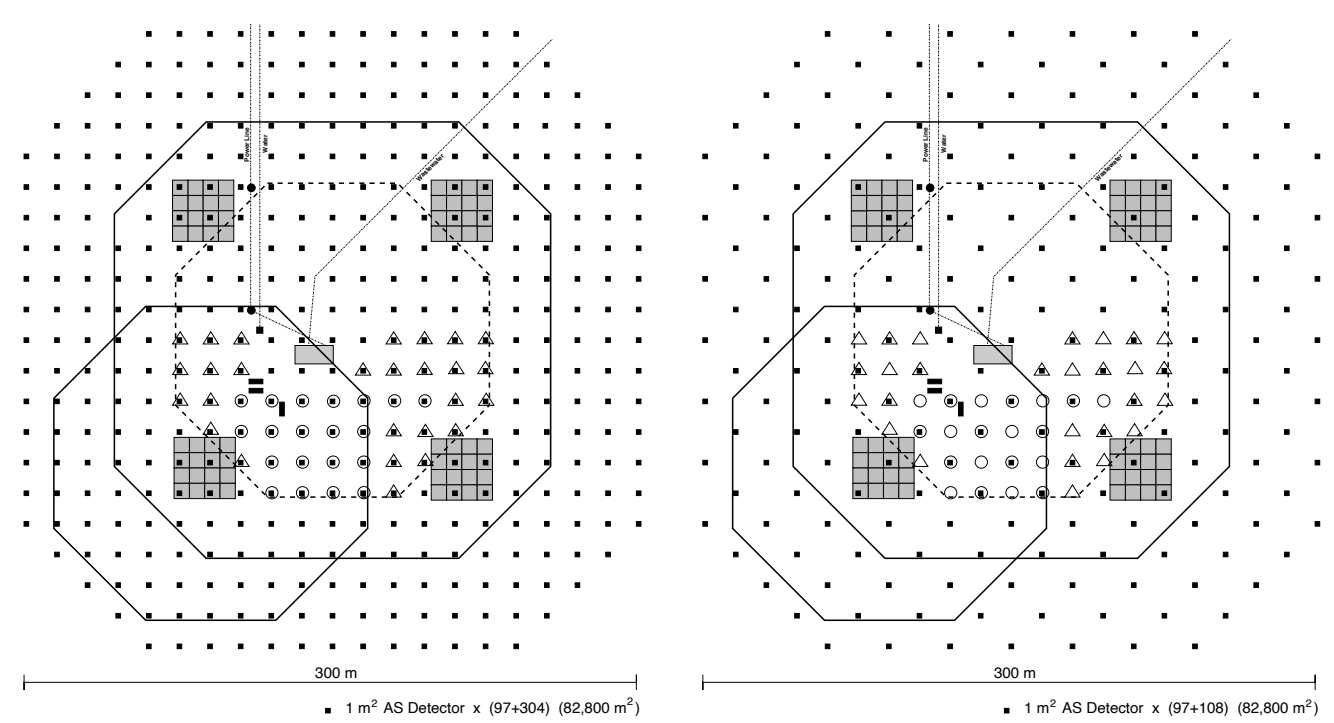

Figure 2: Layouts of ALPACA array at various stages. Small dots indicate SDs and grey hatched areas indicate MDs. (Left) Layout with SDs in $15 \mathrm{~m}$ interval. The enclosed area in the left bottom corner is the ALPAQUITA array under construction. Full coverage array ALPACA (High Density) will be eventually achieved after ALPACA (half). (Right) Layout with SDs in $21 \mathrm{~m}$ interval called ALPACA (half), a stage scheduled after ALPAQUITA.

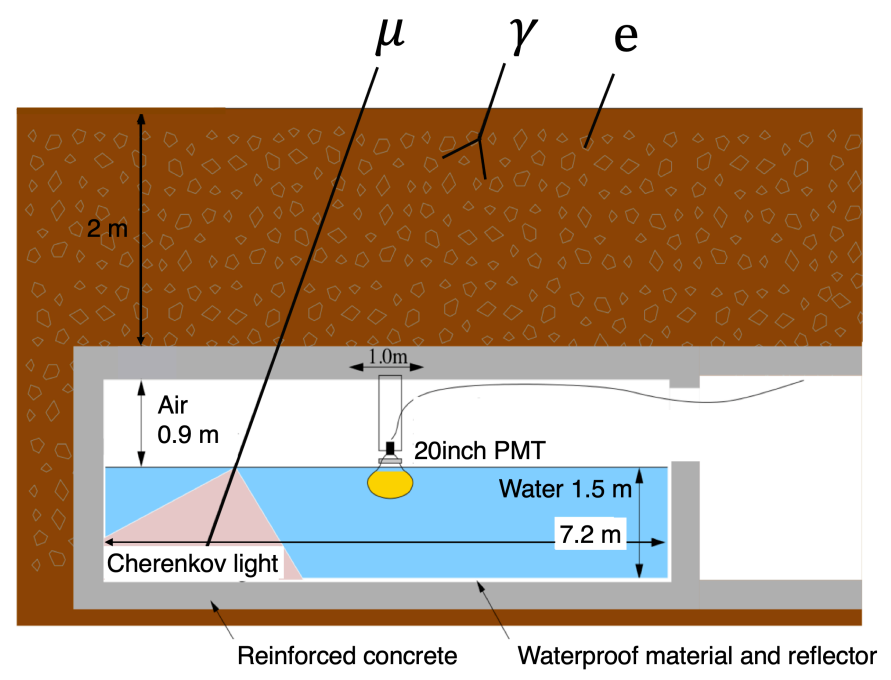

Figure 3: Schematic view of an MD zooming in a single cell. A reinforced concrete structure is constructed under $2 \mathrm{~m}$ soil. MDs are filled with water of $1.5 \mathrm{~m}$ depth and Cherenkov lights in each of $56 \mathrm{~m}^{2}$ cell are monitored by a 20" PMT. 
well of $50 \mathrm{~m}$ depth. At the ALPACA site located on the top of a hill, underground water will exist deeper than the former place. Depending on the depth of the water, the final source of water will be decided. If the water supply is not sufficient, truck transportation from the El Alto town is possible at a reasonable cost.

The design of the first MD is finalized soon, and the MD will be constructed in 2021. In parallel to the MD interior setup (water proof painting, PMT installation, water filling) 97 SDs are arranged as a shape of ALPAQUITA surrounding the MD. Then operation will start as soon as possible.
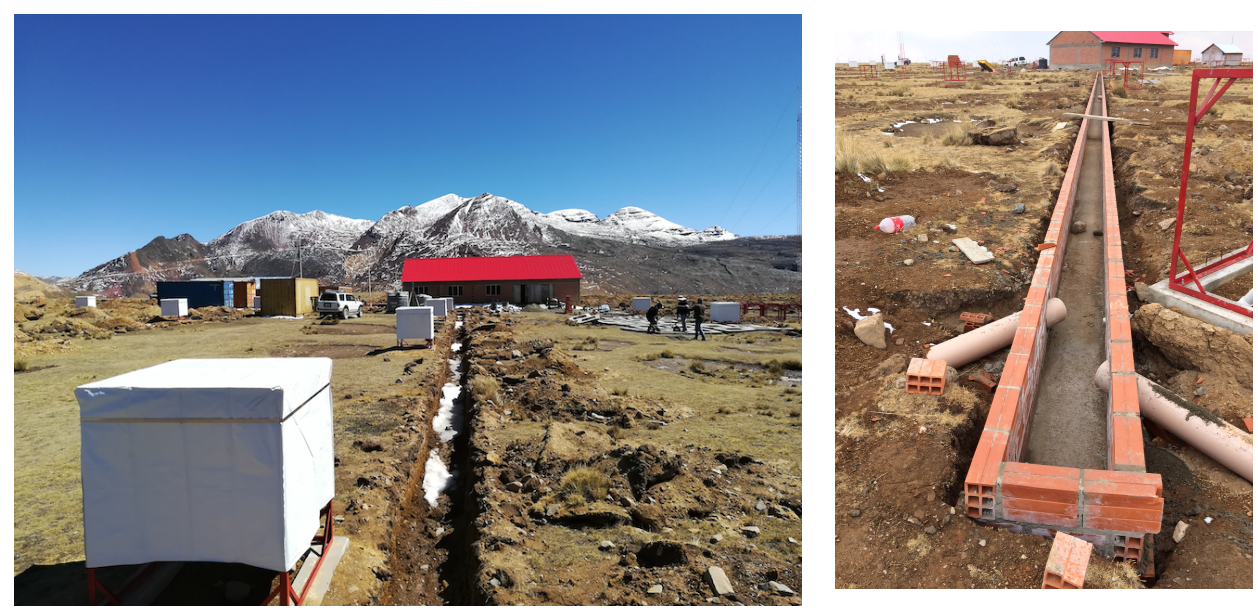

Figure 4: Photos of the ALPACA site. (Left) SDs, central hut and cable drain in preparation. The mountain behind the hut is Chacaltaya. (Right) A cable drain reinforced with bricks and concrete.

\subsection{ALPACA (half) and ALPACA (HD)}

With operating the ALPAQUITA, the construction of the rest 3 MDs starts as seen in Fig.2 (right). Newly prepared 103 SDs and rearranged 97 ALPAQUITA SDs cover a larger area with the detector interval of $21 \mathrm{~m}(\sqrt{2} \times 15 \mathrm{~m})$. Though the detection threshold becomes slightly higher than the ALPAQUITA and ALPACA (HD), this half density ALPACA, aka ALPACA (half), can explore deep in the sub-PeV gamma-ray sky. Expected sensitivity with a dedicated MC study is given in [14]. The gap between SDs will be eventually filled towards ALPACA (HD) to lower the energy threshold and improve energy and angular resolutions.

\section{Beyond PeV - Mega ALPACA}

Beyond the discovery of PeVatrons, further interests are to know the particle acceleration limits in the Galaxy. It is studied by extending the energy spectra both in the individual sources and diffuse gamma rays corresponding to the freshly accelerating objects and acceleration history in the Galaxy. To achieve a necessary sensitivity above $\mathrm{PeV}$ an array with a $1 \mathrm{~km}^{2}\left(1 \mathrm{Mega} \mathrm{m}{ }^{2}\right)$ coverage is required. The idea of Mega ALPACA is proposed as a future extension of ALPACA based on the established technic and experience. The expected sensitivity of Mega ALPACA assuming a $1 \mathrm{~km}^{2}$ surface coverage and 50 MDs is shown in Fig.5. SWGO will improve the sensitivities at the energy 
range covered by ALPACA and lower energy in the future [17]. Mega ALPACA covers higher energy than SWGO complementarily and contributes in a full energy band astronomy.

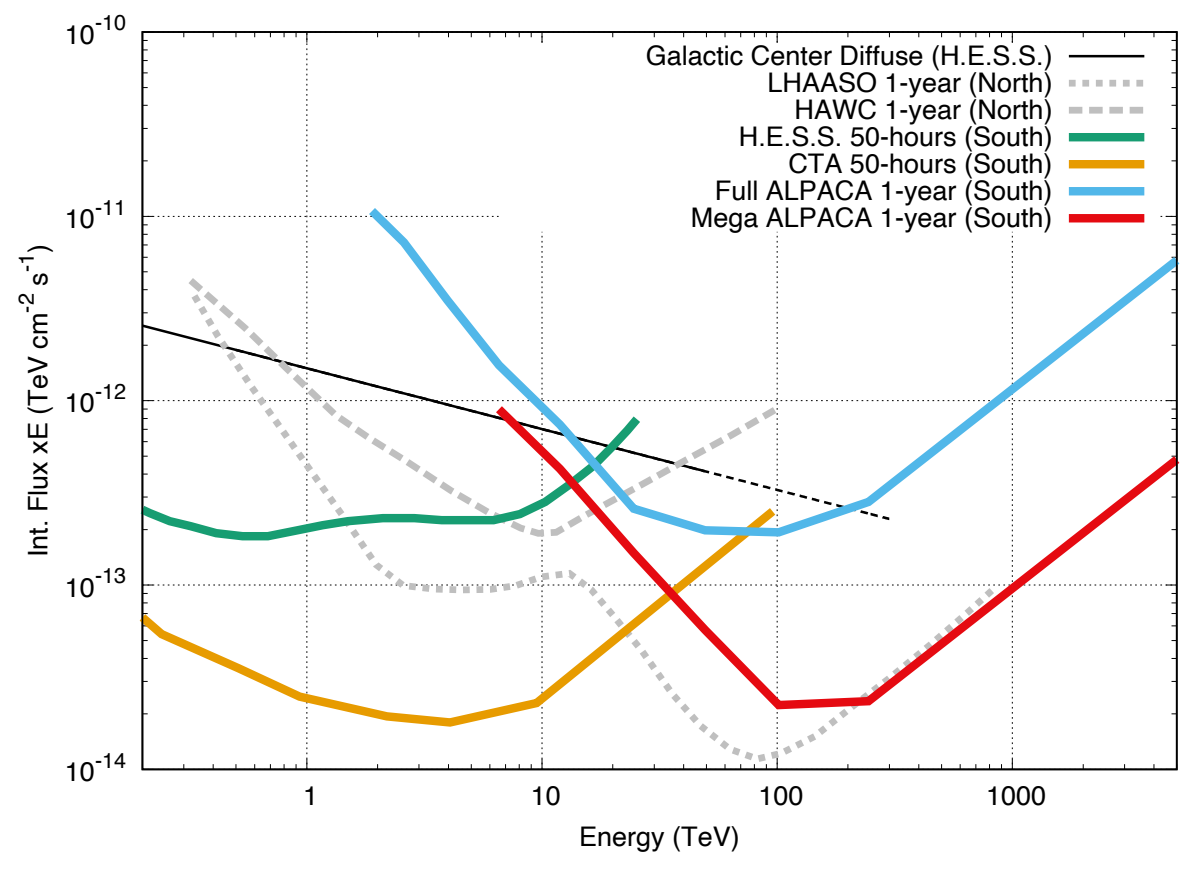

Figure 5: Integral flux sensitivity of Mega ALPACA (red line) together with other gamma-ray observatories in the southern hemisphere (color lines) and in the northern hemisphere (grey lines).

\section{Summary}

Tibet AS $\gamma$, HAWC, and LHAASO opened a new era of astronomy above $100 \mathrm{TeV}$ in the northern hemisphere. Application of these established technics in the southern hemisphere, where richer scientific topics exist, is desired. ALPACA is a project to construct a new air shower array in Bolivia to explore the sub-PeV sky for the first time in the southern hemisphere. The prototype array ALPAQUITA is now under construction and will start an operation in 2021, which will be already sensitive to some bright sources [13]. The infrastructure of ALPAQUITA is ready at the site, and the construction of the first MD will start in the middle of 2021. In parallel to the ALPAQUITA operation, extension to the ALPACA (half) array is scheduled. Extrapolated fluxes of many $\mathrm{TeV}$ sources discovered by the H.E.S.S. galactic plane survey [10] are in the sensitivity of ALPACA (half). The discovery of several sources above $100 \mathrm{TeV}$ is expected, and acceleration limits of individual sources will be discussed. Beyond these discoveries, search for the highest energy accelerators in the Galaxy will be realized by a further extension to Mega ALPACA.

\section{Acknowledgements}

The ALPACA project is supported by the Japan Society for the Promotion of Science (JSPS) through Grants-in-Aid for Scientific Research (A) 19H00678, Scientific Research (B) 19H01922, 
and Scientific Research (S) 20H05640, the LeoAtrox supercomputer located at the facilities of the Centro de Análisis de Datos (CADS), CGSAIT, Universidad de Guadalajara, México, and by the joint research program of the Institute for Cosmic Ray Research (ICRR), The University of Tokyo. K. Kawata is supported by the Toray Science Foundation. E. de la Fuente thanks Coordinación General Académica y de Innovación (CGAI-UDG), cuerpo académico PRODEPUDG-CA-499, Carlos Iván Moreno, Cynthia Ruano, Rosario Cedano, and Diana Naylleli, for financial and administrative support during sabbatical year stay at the ICRR on 2021. I. ToledanoJuarez acknowledges support from CONACyT, México; grant 754851.

\section{References}

[1] The Tibet AS $\gamma$ Collaboration, Phys. Rev. Lett., 123, 051101 (2019).

[2] The HAWC Collaboration, Astrophys. J., 881:134 (2019).

[3] The LHAASO Collboration, Chinese Phys. C, 45, 025002 (2021).

[4] A. U. Abeysekara, A. Albert, R. Alfaro et al., Nature Astronomy 5, 465-471 (2021).

[5] The Tibet AS $\gamma$ Collaboration, Nature Astronomy 5, 460-464 (2021).

[6] Z. Cao, F. A. Aharonian, Q. An et al., Nature, 594, 33-36 (2021).

[7] The Tibet AS $\gamma$ Collaboration, Phys. Rev. Lett., 126, 141101 (2021).

[8] K. Fang and K. Murase, arXiv:2104.09491 (2021).

[9] B.-Q. Qiao, W. Liu, M.-H. Zhao, X.-J. Bi and Y.-Q Guo, arXiv:2104.03729 (2021).

[10] The HESS Collaboration, A\&A 612, A1 (2018).

[11] The HESS Collaboration, Nature, 531, 476-479 (2016).

[12] P. Lipari and S. Vernetto, Phys. Rev. D 98, 043003 (2018)

[13] S. Kato et al. (The ALPACA Collaboration), PoS (ICRC2021) 857.

[14] Y. Yokoe et al. (The ALPACA Collaboration), PoS (ICRC2021) 947.

[15] T. K. Sako, K. Kawata, M. Ohnishi, A. Shiomi, M. Takita, H. Tsuchiya, Astropart. Phys. 32, 177-184 (2009).

[16] The Tibet AS $\gamma$ Collaboration, Astrophys. J., 813:98 (2015).

[17] P. Huentemeyer et al., Astro2020 APC White Paper (2019).; arXiv:1907.0773 (2019). 


\section{Full Authors List: ALPACA Collaboration}

F. Aceves de la Cruz ${ }^{1}$, S. Asano ${ }^{2}$, C. A. H. Condori ${ }^{3}$, E. de la Fuente ${ }^{1,4}$, J. L. Garcia-Luna ${ }^{1}$, A. Gomi ${ }^{5}$, K. Hibino ${ }^{6}$, N. Hotta ${ }^{7}$, A. Jimenez-Meza ${ }^{8}$, Y. Katayose ${ }^{9}$, C. Kato ${ }^{2}$, S. Kato ${ }^{10}$, T. Kawashima ${ }^{10}$, K. Kawata ${ }^{10}$, T. Koi ${ }^{11}$, H. Kojima ${ }^{12}$, D. Kurashige ${ }^{5}$, J. Lozoya ${ }^{13}$, R. Mayta ${ }^{14,15}$, P. Miranda ${ }^{3}$, K. Munakata ${ }^{2}$, K. Nagaya ${ }^{5}$, Y. Nakamura ${ }^{10}$, Y. Nakazawa ${ }^{16}$, C. Nina ${ }^{3}$, M. Nishizawa ${ }^{17}$, S. Ogio ${ }^{14,15}$, M. Ohnishi ${ }^{10}$, S. Okukawa ${ }^{5}$, F. Orozco ${ }^{4}$, A. Oshima ${ }^{11}$, M. Raljevich ${ }^{3}$, H. Rivera ${ }^{3}$, T. Saito ${ }^{18}$, Y. Sakakibara ${ }^{5}$, T. Sako ${ }^{10}$ T. K. Sako ${ }^{10}$, S. Shibata ${ }^{12}$, A. Shiomi ${ }^{16}$, M. Subieta ${ }^{3}$, N. Tajima ${ }^{19}$, W. Takano ${ }^{6}$, M. Takita ${ }^{10}$, Y. Tameda ${ }^{20}$, K. Tanaka ${ }^{21}$, R. Ticona ${ }^{3}$, I. Toledano-Juarez ${ }^{22,23}, \mathrm{H}$. Tsuchiya ${ }^{24}$, Y. Tsunesada ${ }^{14,15}, \mathrm{~S}$. Udo ${ }^{6}, \mathrm{~K}$. Yamazaki ${ }^{11}$ and Y. Yokoe ${ }^{10}$

${ }^{1}$ Departamento de Física, CUCEI, Universidad de Guadalajara, Guadalajara, Jalisco, Mexico. ${ }^{2}$ Department of Physics, Shinshu University, Matsumoto, Nagano 390-8621, Japan. ${ }^{3}$ Instituto de Investigaciones Físicas, Universidad Mayor de San Andrés, La Paz 8635, Bolivia. ${ }^{4}$ Doctorado en Tecnologías de la Información, CUCEA, Universidad de Guadalajara, Zapopan, Jalisco, Mexico. ${ }^{5}$ Graduate School of Engineering Science, Yokohama National University, Yokohama, Kanagawa 240-8501, Japan. ${ }^{6}$ Faculty of Engineering, Kanagawa University, Yokohama, Kanagawa 221-8686, Japan. ${ }^{7}$ Utsunomiya University, Utsunomiya, Tochigi $321-$ 8505, Japan. ${ }^{8}$ Departamento de Tecnologíias de la Información, CUCEA, Universidad de Guadalajara, Zapopan, Jalisco, Mexico. ${ }^{9}$ Faculty of Engineering, Yokohama National University, Yokohama, Kanagawa 240-8501, Japan. ${ }^{10}$ Institute for Cosmic Ray Research, University of Tokyo, Kashiwa, Chiba 277-8582, Japan. ${ }^{11}$ College of Engineering, Chubu University, Kasugai, Aichi 487-8501, Japan. ${ }^{12}$ Chubu Innovative Astronomical Observatory, Chubu University, Kasugai, Aichi 487-8501, Japan. ${ }^{13}$ Departamento de Ciencias de la Información y Desarrollo Tecnológico, Cutonala, Universidad de Guadalajara, Tonalá, Jalisco, Mexico. ${ }^{14}$ Graduate School of Science, Osaka City University, Osaka, Osaka 558-8585, Japan. ${ }^{15} \mathrm{Nambu}$ Yoichiro Institute for Theoretical and Experimental Physics, Osaka City University, Osaka, Osaka 558-8585, Japan. ${ }^{16}$ College of Industrial Technology, Nihon University, Narashino, Chiba 275-8576, Japan. ${ }^{17}$ National Institute of Informatics, Chiyoda, Tokyo 101-8430, Japan. ${ }^{18}$ Tokyo Metropolitan College of Industrial Technology, Arakawa, Tokyo 116-8523, Japan. ${ }^{19}$ RIKEN, Wako, Saitama 351-0198, Japan. ${ }^{20}$ Faculty of Engineering, Osaka ElectroCommunication University, Neyagawa, Osaka 572-8530, Japan. ${ }^{21}$ Graduate School of Information Sciences, Hiroshima City University, Hiroshima, Hiroshima 731-3194, Japan. ${ }^{22}$ Doctorado en Ciencias Físicas, CUCEI, Universidad de Guadalajara, Guadalajara, Jalisco, Mexico. ${ }^{23}$ Maestría en Ciencia de Datos, Departamento de Métodos Cuantitativos, CUCEA, Universidad de Guadalajara, Zapopan, Jalisco, Mexico. ${ }^{24}$ Japan Atomic Energy Agency, Tokai-mura, Ibaraki 319-1195, Japan. 